\title{
Modeling of the Catalysts Behavior for Determination of Bordering Conditions of Special Properties of Nanocomposites
}

\author{
Mukhamediev MG* and Rakhimov T Kh \\ Department of Polymer Chemistry, National University of Uzbekistan, 4 Universitet Ko'chasi, Tashkent 100174, Uzbekistan
}

Received: February 16, 2015; Accepted: April 30, 2015; Published: May 14, 2015

*Corresponding author: Mukhamediev MG, Department of Polymer Chemistry, National University of Uzbekistan, 4 Universitet Ko'chasi, Tashkent 100174, Uzbekistan, Tel: +998-93-5704136; Fax: +998-71-144-73-12; E-mail:-mmuxamediev@mail.ru

\begin{abstract}
The paper is devoted to the determination of active nanoparticles sizes. Other particles that deviate from the calculated size limits do not demonstrate the special activity that is the catalytic elimination of environmental pollutants, namely, carbon monoxide, under ambient conditions - low temperature, high humidity and the presence of anthropotoxins. This paper discusses the basic principles of catalysts used in environmental pollution abatement, in particular, the effect of nanocatalyst size on catalytic activity. It remains unclear why large palladium containing objects, as well as true solutions are not effective as catalysts for low-temperature carbon monoxide oxidation. Additionally it is know that only palladium containing nano systems over a narrow size range are effective catalysts. The submitted work is the first step in quantifying this range. Hopefully this will assist in the determination of the causes of unusually high nanocatalysts efficiency.
\end{abstract}

Keywords: Nano Particles; Nano Crystalline; Mathematical Modeling; Nano Catalysts; Critical Grain Size

\section{Introduction}

At present, the level of theoretical understanding of the structure and properties of compact nanocrystalline materials is much lower compared with the available theoretical models of the structure and properties of various isolated nanoparticles [1]. The properties of bulk nanomaterials in relation to the particle size were discussed in reviews [2,3]. This influence most clearly manifested at catalysis.

Usually, the nanoparticles show catalytic activity in a very narrow size range [4-6]. Unique nanoparticles' properties naturally occur if the particle size does not exceed a certain value [7]. Obviously, this is also due to surface phenomena. However, not all of the effects can be explained by in surface area increasing. For example if the atoms located on the faces are catalytically active, the rate of the catalyzed reaction will be increased by larger particles [8,9]. The main question remains on how to determine the upper and sometimes the lower limits of the sizes in which nano-properties appears. These borders could be determined by experiments with the detection of the size effect. Such experiment should be carried out on materials of the same chemical composition but different dispersion in grain size. The important condition of this experiment is equal size of the particles or grains of material investigated. Unfortunately, at the present time it is not possible to carry out such experiments [10].

We believe that these limits can be calculated using the tools of mathematical statistics on the basis of experimental data obtained on materials with significant dispersion of particle sizes. Submitted paper describes the principles of such approach.

\section{Experimental}

\section{Objects of investigations}

Drawing catalysts comprising platinum metals - palladium and platinum - as active ingredient has been investigated. Fiber polymers - polypropylene fiber polyacrylonitrile, carbon fibers, low modulus carbon fibrous materials obtained by carbonization and subsequent activation of mats from "Mtilon-M", copolymers of polyacrylonitrile and hydrocellulose were used as bearers.

\section{Subjects and methods}

Catalytic activity is dependent on CO concentration in the gas-air mixture at room conditions [11]. The rms-diameter and coefficient of polydispersionality of nano-particles [12] were measured via electronic microscopy.

\section{Results}

\section{Catalytic activity as special nanoparticles property}

With the advent of nanocatalysts, exhaustive removal of toxic components (such as $\mathrm{CO}$ ) from air by passive systems is now possible $[13,14]$. The influence of the substrate's nature on the activity and stability of the catalytic process has been determined [15]. At the same time it is obvious the physical and chemical parameters of the active component will exert not less influence on the properties of the catalytic system as a whole.

In this vein, one of the most important characteristics of nanoparticles is their dimensions. In terms of chemical properties 
of nanoparticles the most important characteristics aren't their linear dimensions, weight or volume but their surface area.

The quantitative statistical characteristics used to reflect the average relative values of the active surface of nanomaterials are the rms diameter and polydispersity index of the nanoparticles [12]. The question has arisen: from which dimension of the particles some special properties inheriting namely nanostructures have appeared and at what dimensions they have disappeared? Which features are necessary for a material to be considered a nanomaterial?

For investigation of influence of qualitative changing of properties of nano-composites it is purposefully to turn the most evident manifestations peculiarities inherent exceptionally to nano-particles. To such teases can be attributed effects shown by drawing palladium nano-catalysts of low-temperature oxidation of $\mathrm{CO}$ by oxygen. Really exhaustive removal of $\mathrm{CO}$ by its catalytic oxidation by oxygen of air at room conditions don't note neither for too small (solutions, solid solutions) nor too large (massive samples) objects of similar chemical compositions.

In composition of investigated high-active nano-catalysts drawing on polymeric bearers as inorganic and organic nature the active components has presented as particles of complex composition with dimensions 10-1000 nm [14] containing platinum metals and their compounds. More clearly there particles can be examine on the microphotographs of samples with carbon fibrous materials as bearers (Figure 1). There is a rather high level of the dispersion of the size of the particles.

\section{Are there any exclusive properties of nanoparticles}

Before construction of model it is necessary to convince in fact: there are or not some qualitative, spasmodic changes in properties inherited to substances characterized exceptionally by nano dimensions. If such changes have absented then only quantitative interdependence of smooth changes of properties with parameter connecting with dimensions of particles must be presented.

Let us proposed that at display of high catalytic activity of composites special effects connected with nano-dimensions absent. Then in rate of catalytic heterogeneous reactions the role of the summary area of surface of all particles which are accessible to substrates will be very important. In such case it is possible to expect that catalytic activity will be increase smoothly with increasing of total area of surface. Even if this connection isn't linear never the less it must not be spasmodic on some segment of curve. Proceed from supposition about spherical of particles their volume is proportional to cube of radius and the area of surface - to quadrat of ball diameter. Proceeding from ball with radius $d^{*} \mathrm{R} d 3$ balls with less radius $\mathrm{R}$ will be obtain it is easily to understand that the total area of surface with decreasing of radius in " $d$ " times will increased at the same times.

Comparison of catalytic activity of samples with palladium on the fiber obtained by carbonization and following activation of Mtilon-M [16] has shown that there is sharp increasing of activity for nano-particles with dimensions 300-1000 nm (Table 1). The same qualitative changing of catalytic activity was observed for samples on the base of carbonized hydrocellulose with dimensions of particle 40-100 $\mathrm{nm}$ (area of active surface 300 $\left.\mathrm{m}^{2} / \mathrm{g}\right)$.

These data have witnessed in benefit of hypothesis about presence of brightly expressed effect of increasing of catalytic activity at increasing of content of nano-particles of definite radiuses. Apparently due to the marked sizes of particles with

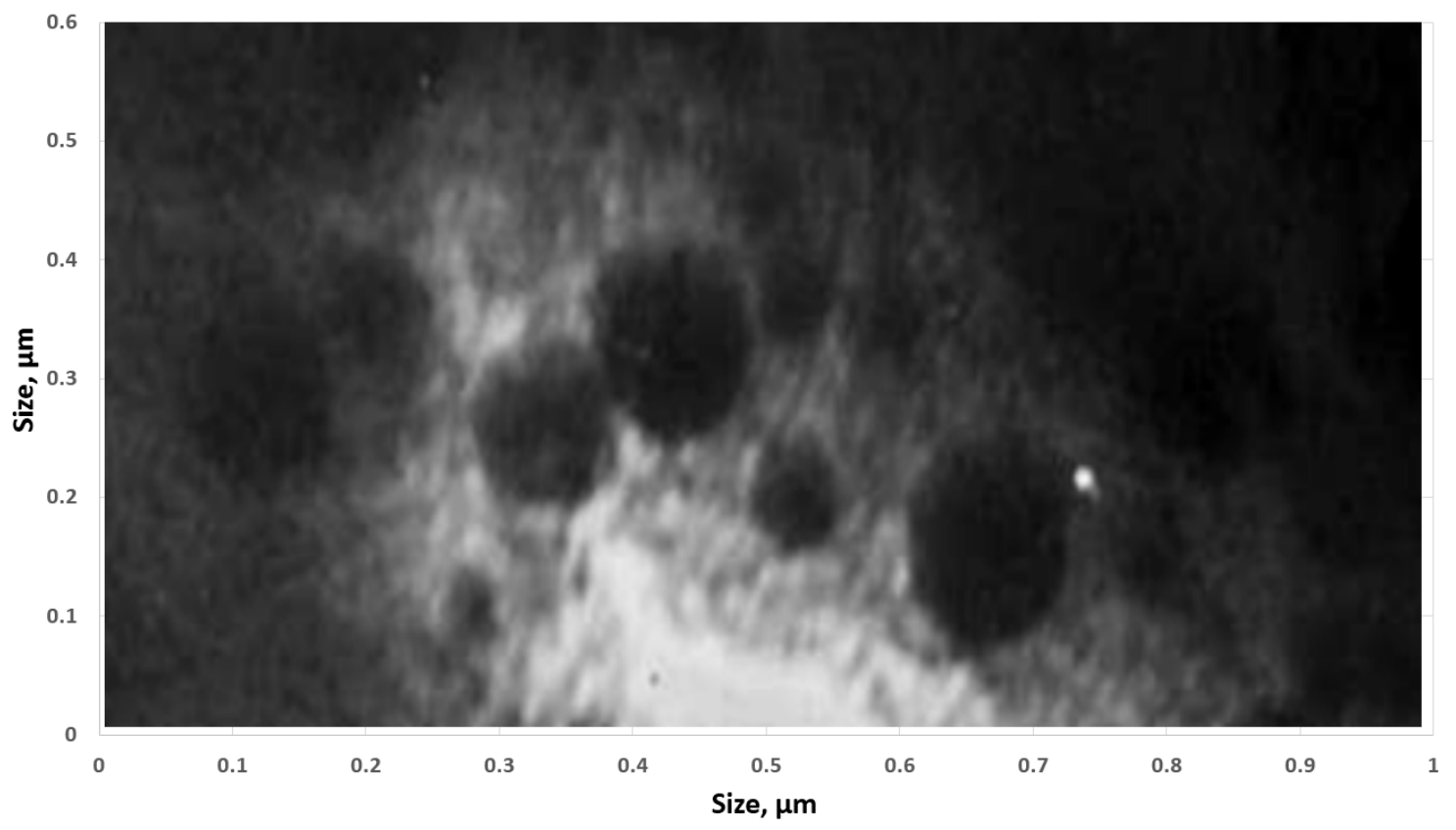

Figure 1: Microphotography of activated carbon fiber obtained by carbonization of Mtilon-M with drawing palladium. 
Table 1: Activity of palladium-containing nano-catalysts (4 mass \%) with different average squared diameters of particles. In the following regime, bold italics are attributed to data on the base of which calculations were carried out.

\begin{tabular}{|c|c|c|c|}
\hline $\begin{array}{l}\text { Average squared } \\
\text { diameter, } \mathrm{nm}\end{array}$ & $\begin{array}{c}\text { Specific activity*10 } \\
l^{*} \mathbf{s}^{*} \mathrm{~g}\end{array}$ & $\begin{array}{l}\text { Increasing of total surface } \\
\text { in times }\end{array}$ & $\begin{array}{l}\text { Increasing of specific activity in calculation on the } \\
\text { unit of area of surface in times }\end{array}$ \\
\hline \multicolumn{4}{|c|}{ Bearer - activated carbon fibrous materials from Mtilon-M, active surface area $2700 \mathrm{~m}^{2} / \mathrm{g}$} \\
\hline 2000 & 0.2 & 1 & 1 \\
\hline 1500 & 1.4 & 1.3 & 5 \\
\hline 1200 & 1.12 & 1.7 & 3 \\
\hline 1000 & 15.6 & 2 & 39 \\
\hline 800 & 22.1 & 2.5 & 44 \\
\hline 720 & 21.1 & 2.8 & 38 \\
\hline 601 & 24 & 3.3 & 36 \\
\hline 511 & 27.5 & 3.9 & 35 \\
\hline 380 & 15.1 & 5.3 & 14 \\
\hline 222 & 6.4 & 9 & 4 \\
\hline 104 & 2.9 & 19.2 & 0.8 \\
\hline 62 & 0.8 & 32.3 & 0.1 \\
\hline 47 & 0.2 & 42.6 & 0 \\
\hline 24 & 0 & 83.3 & 0 \\
\hline 12 & 0 & 166.7 & 0 \\
\hline \multicolumn{4}{|c|}{ Bearer -carbon fibrous materials from hydrocellulose, active surface area $300 \mathrm{~m}^{2} / \mathrm{g}$} \\
\hline 200 & 0 & & \\
\hline 120 & 0.11 & 1 & 1 \\
\hline 111 & 0.09 & 1.1 & 0.8 \\
\hline 98 & 0.84 & 1.2 & 6.2 \\
\hline 85 & 1.4 & 1.4 & 9 \\
\hline 77 & 1.12 & 1.6 & 6.5 \\
\hline 64 & 1.16 & 1.9 & 5.6 \\
\hline 54 & 1.21 & 2.2 & 5 \\
\hline 44 & 1.21 & 2.7 & 4 \\
\hline 36 & 0.41 & 3.3 & 1.1 \\
\hline 28 & 0.27 & 4.3 & 0.6 \\
\hline 19 & 0.12 & 6.3 & 0.2 \\
\hline 10 & 0 & 12 & 0 \\
\hline Less than 10 & 0 & 24 & 0 \\
\hline
\end{tabular}

a more or less deviations from average responsible for catalytic activity are nano-structures with more narrow limits of particles dimensions.

On the base of obtained data it is shown that effect of spasmodic increasing activity for composites with definite average dimensions of particles can't be explained by increasing of the total surface of contact of particles with substrate. Thus it is determined that there are effects caused by nano-dimensions of composite's particles.

\section{Bordering conditions of exclusive properties of nanocatalysts}

Logically the second question is raised - from what dimension of particles the nano-properties have appeared and at what minimal dimension of them they have ended? With others words - how to determine those limits at which particles can be named as nanoparticles and containing them composites nanocomposites? It is clearly that for each system these values will be different.

Estimation of limits of minimal and maximal dimensions of nanoparticles outside of which "nano properties" have disappeared is impossible without mathematical approaches. For statement of above-mentioned task the evident model is proposed which has lightened the understanding of chemical problems by mathematicians. This model conditionally was 
named "model of balls coloring". Lower the description of this model is cited.

\section{Model of balls coloring}

\section{Description of task:}

Hypothetical case: It is necessary to paint by different colors the surface of balls which in reality are ellipsoids stretching in one direction, but in first approaching we shall suppose them as balls. Color in this case will be corresponded to definite experimental sample possessed by measured macro-properties - in our case it is correspondently nano-catalysts and their catalytic activity. Ball were in some containers. In each container there are enough many balls - about 10 milliards and by this reason the statistical regularities can be used. The common volume (or mass) of all balls for all containers is similar (naturally, the poured volume of balls will be different, but this fact isn't important). Balls from each container must be painted in the same color but from different containers - in different colors.

However not all balls must be colored. It is decided no paint too small and too large balls. There are known different and limited amounts of dyes for each color. Expenditure of dye for the unit of surface of balls is the same. It is obvious that these amount from the point of view of chemist will corresponded to observed and measurable macro-property - in our case to catalytic activity determined as rate of oxidation reaction of $\mathrm{CO}$ in the presence of given concrete sample of catalyst.

Required to determine: It is necessary to select such values of minimal and maximal radius of balls that dye will spend optimally.

Measured and available data: Before carrying out of calculations from each container not less than 40 balls have been selected randomly and their radiuses were measured. In result for each container two characteristics were obtained: average squared radius of ball which was calculated on the base of experimental results by formula:

$$
\bar{R}=\sqrt{\frac{\sum_{i=1}^{n} R_{i}^{2}}{n}}
$$

And the coefficient of polydispersion $\mathrm{L}$ - which is indifferent value (expressed in \%) and has characterized an irregularity of nanoparticles dimensions. This parameter was calculated on the base of experimental data by formula:

$$
L=\frac{\sum_{i=1}^{n}\left(\bar{R}-R_{i}\right)^{2}}{n \bar{R}^{2}}
$$

Quantity of dye (in given case it has corresponded to rate of reaction in the presence of given sample) was known before hand (in given case this quantity was obtained in results of experiments on the base of measuring of catalytic activity that is by decreasing of $\mathrm{CO}$ concentration on the outlet).

As example data obtained on the base of real measures by rate determination $(\mathrm{V})$ of catalytic reaction for activated carbon fibers on the base of Mtilon-M with drawing nanoparticles of palladium are presented below (Table 2). For giving clearness to the mathematical problem values "nm" were substituted for " $\mathrm{mm}$ " and V - for volume in $\mathrm{m} 3$.

Also the function of balls distribution by their dimensions is necessary for carrying out of above-mentioned calculation. We have supposed that this distribution is normal - Gaussian distribution.

\section{Principles of calculation}

It is necessary to introduce values of minimal $m$ and maximal $M$ radiuses outside of which coloring didn't occur. For finding of necessary quantity of dye it is necessary to calculate the total area of surface of colored balls.

For each container values of the total area of surface of balls were calculated. For each i-ball:

$$
\mathrm{Si}=4 \pi \mathrm{Ri} 2
$$

If radiuses of balls have changed on small value $\mathrm{dR}$, than for determination of the total area of balls surface it is necessary to calculate some determined integral:

$$
s=\int_{m}^{M} 4 \pi f\left(R^{2}\right) d R
$$

Function $f\left(R^{2}\right)$ is constructed by analytically on the base of data about type of distribution of balls by dimensions - Gaussian distribution; values of their average dimension and coefficient of polydispersity.

In result we have elaborated analytical method of determination of the total area of surface of all painted balls from each container in dependence on given values of " $\mathrm{m}$ " and " $\mathrm{M}$ " (Figure 2).

Determination of average squared deviation (error): After calculation of analytically common surface of dying balls for each container dependence of calculated and factual amount of dye was constructed. Deviations were determined by method of differentiation (for example, by method of least squares). Given values of " $\mathrm{m}$ " and " $\mathrm{M}$ " it is possible to find values of error.

Table 2: Data to build model of balls coloring. Data compiled on the basis of experimental data on the measurement of catalytic activity of activated carbon fibers on the base of Mtilon-M with drawing nanoparticles of palladium.

\begin{tabular}{|l|c|c|c|}
\hline \multicolumn{1}{|c|}{$\begin{array}{c}\text { Color of painted } \\
\text { balls }\end{array}$} & $\begin{array}{c}\text { Average squared radius, } \\
\mathbf{m m}\end{array}$ & $\mathbf{L , ~ \%}$ & Volume, $\mathbf{~ m}^{\mathbf{3}}$ \\
\hline Red & 125 & 144 & 1.12 \\
\hline Orange & 263 & 90 & 1.28 \\
\hline Yellow & 224 & 98 & 1.92 \\
\hline Green & 336 & 60 & 2.56 \\
\hline Blue & 301 & 71 & 3.28 \\
\hline Dark blue & 222 & 95 & 4.48 \\
\hline Violet & 336 & 57 & 4.8 \\
\hline Brown & 349 & 49 & 9.84 \\
\hline White & 268 & 38 & 22 \\
\hline Black & 6 & 81 & 0.08 \\
\hline
\end{tabular}




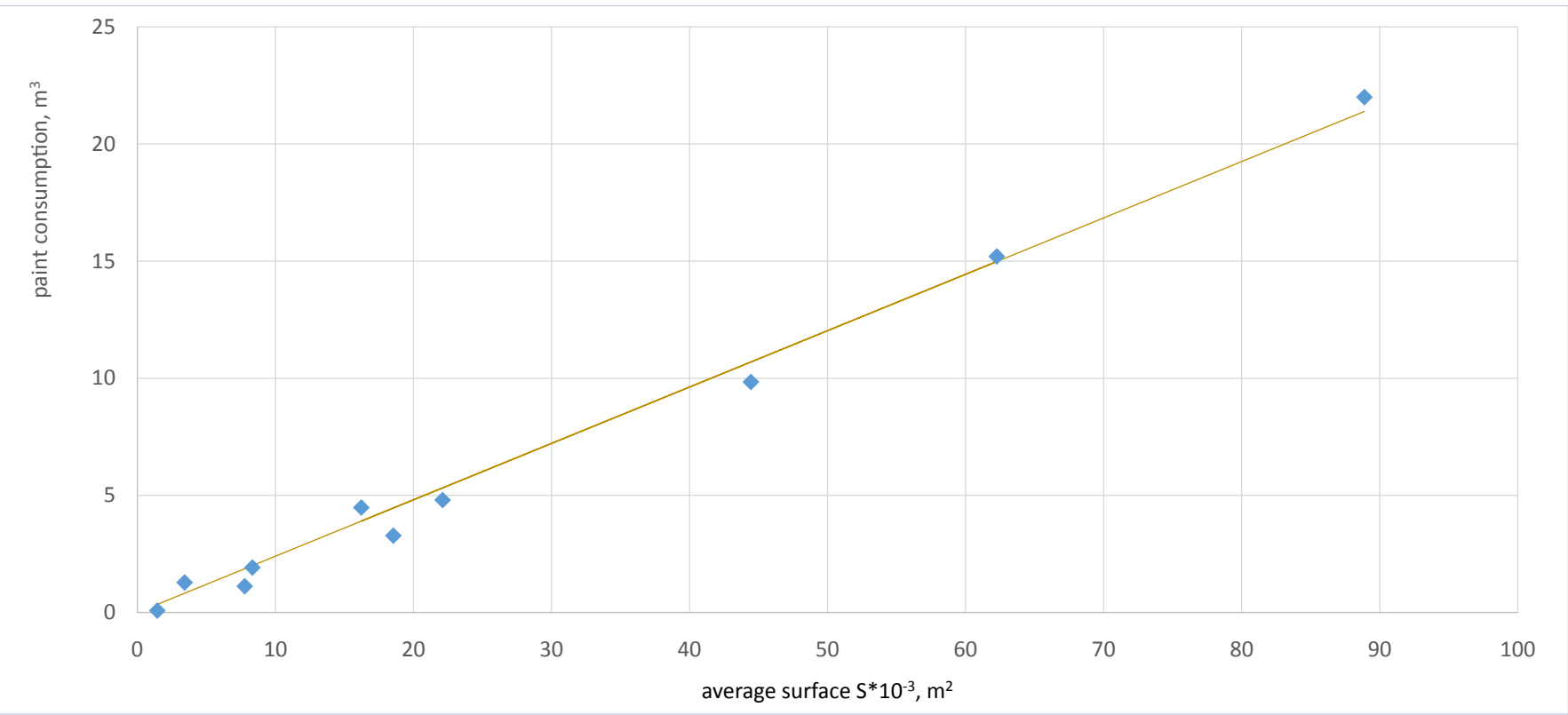

Figure 2: Total surface of painted balls. Regression coefficient 0.24 .

Also for higher clearness it is possible to make following conclusion "the more in this container balls of average dimension the more dye is required. And this fact also is depended on average dimension of balls, type of distribution and values of polydispersion - the more evenly dimensions of balls have distributed around an average value the more dye will be demanded and also disperseness of eliminated for dying balls will be narrow.

Then formula of dependence value of deviation (confidential interval) at fixed probability for given values " $m$ " and "M" was determined. This formula will be final formula for our model.

Determination of values of minimal and maximal radiuses: Now there is formula by which it is possible to find values of the confidential interval $\mathrm{X}$ or average squared deviations. By given values of " $m$ " and " $M$ " in this formula sought for values of boundaries of interval of balls dimensions will be supposed those for which confidential interval is minimum that is there is coincidence of calculated values with experimental data (Figure 3).

These values can be obtained by following method. At given step for " $m$ " and "M" X can be calculated. Geometrically this will be the surface where " $\mathrm{m}$ " and "M" will be correspondently abscissa and ordinate, and $X$ - applicate. Minimum of this surface is determined and then values of " $\mathrm{m}$ " and " $\mathrm{M}$ " can be calculated

Using and development of model: For concrete nanosystems (in our case - nano-catalysts) values of " $m$ " and " $M$ " have allowed to give nanostructures the quantitative determination and determine real borders inside of which the special properties have displayed.

At the same time the proposed model don't take into account some factors which can be very considerable. Properties of nanostructures in particular catalytic activity can be depended on some parameters (in our case - from area of surface) not linearly: form of nanoparticles can differed from spherical or ellipsoidal shape; structure and chemical properties can differ for particles of different dimensions and also in limits of one particle. If proposed approach will be fruitful than this model will be needed in some précises and development.

\section{Conclusion}

Simple proofs are cited about qualitative differences of properties of nanoparticles from objects dimensions of which are behind limits nano-dimensions. On the example of higheffective polymeric nanocatalysts of low temperature oxidation of $\mathrm{CO}$ in such nano-composites the critical function is a content of particles of active component with definite dimensions values of which are inside clearly designated limits values of which are different for different systems. Limits inside of the borders have determined qualitative peculiarity of nanostructures properties.

Mathematical model was proposed which has allowed determining limits of nanoparticles dimensions in borders of which there have displayed some special properties inherited to only nanostructures. The model has allowed obtaining decisions, that is to find values of maximum and minimum radiuses of particles in particular for catalytically active nanocomposites and also can be expended for exposure of others special properties inherited only to nano-systems.

Described method allows finding the answer to one of two questions that's consist the main scope of investigations of the nanocrystalline state: is there some critical grain (particle) size below which the characteristic properties of nanocrystals become observable, and above which the material behaves as a bulk one. 


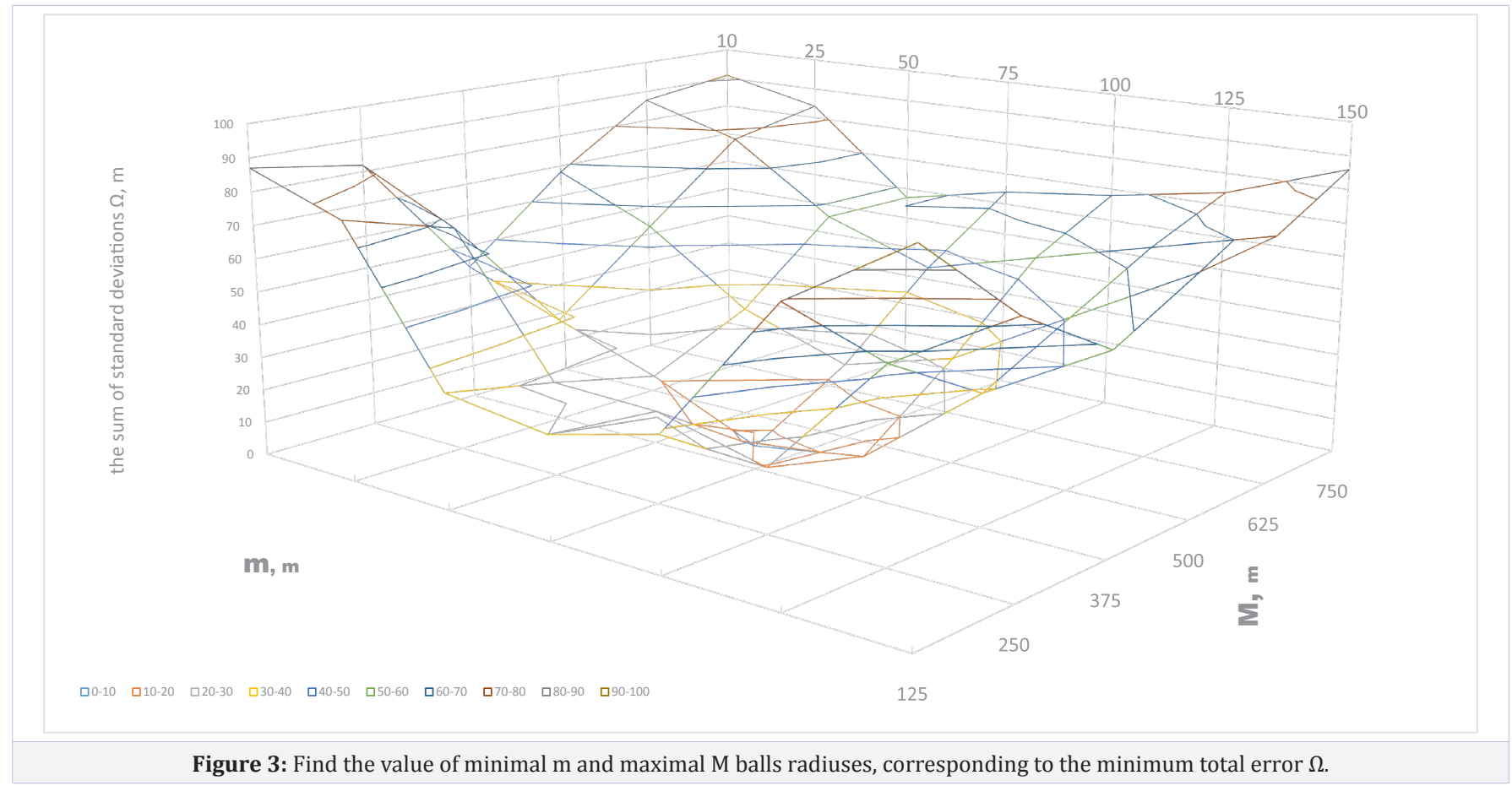

\section{References}

1. Gusev AI, Rempel AA. Nanocrystalline Materials. Cambridge: Cambridge International Science Publishing; 2004.

2. Gusev AI. Effects of the nanocrystalline state in solids. Uspekhi Fiz. Nauk. 1998; 41: 49-76.

3. Andrievski RA, Glezer AM. Size effects in nanocrystalline materials: I. Structure characteristics, thermodynamics, phase equilibria, and transport phenomena. Fiz. Metal. Metalloved. 1999; 88, No 1: 50-73 (in Russian).

4. Che M., Bennet CO. The influence of particle size on the catalytic properties of supported metals. Advances in Catalysis. 1989; 36: 55172.

5. Ponec V. Catalysis by alloys in hydrocarbon reactions. Advances in Catalysis. 1983; 32: 149-214.

6. Karpinski Z. Catalysis by supported, unsupported, and electrondeficient palladium. Advances in Catalysis. 1990; 37: 45-100.

7. Briot P, Auronx A, Jones D, Primet M. Effect of particle size on the reactivity of oxygen-absorbed platinum supported on alumina. Appl. Catalysis. 1990; V.59: P. 141-152.

8. Bukhtiyarov VI, Slinko MG. Metallic nanosystems in catalysis. Russian Chemical Reviews. 2001; v. 70, n.2: 147-159.

9. Goodman DW. Catalysis: from single crystals to the "real world". Surface Sci. 1994; V.299/300: P.837-848.

10. Gusev AI. Nanomaterials, Nanostructures, and Nanotechnologies. Moscow: Fizmatlit; 2005, p. 21.
11. Rakhimov TKh, Mukhamediev MG. Simultaneous gas chromatographic determination of carbon oxides in the environment. $4^{\text {th }}$ Republican scientific and engineering conference "Actual problems of analytical chemistry", Termez, May 1-3, 2014. Book Of Abstracts, Part 2: p. 277 278.

12. Rakhimov TKh. Quantitative Criteria For The Comparative Size Of The Nanoparticles. $8^{\text {th }}$ International Symposium "Molecular Order and Mobility in Polymer Systems", St. Petersburg, June 2-6, 2014. Book Of Abstracts: 0-42.

13. Shuth P, Song X, Shmidt LD, Wicke E. Synchrony and The Emergence of Chaos in Oscillation on Supported Catalysts. J. Chem. Phys. 1990; 92, N 1: p.745-756.

14. Rakhimov TKh, Mukhamediev MG, Khakimjanov BSh. The Synthesis Of Applied Nano-Catalysts Of Low-Temperature Carbon Monoxide Oxidation. 8th International Symposium "Molecular Order and Mobility in Polymer Systems", St. Petersburg, June 2-6, 2014. Book Of Abstracts: P-116.

15. Rakhimov TKh, Mukhamediev MG. Palladium Containing Composite Nanosystems In Low-Temperature Co Oxidation: Decisive Influence Of The Substrate To The Reactions' Mode. Composite Materials Uzbekistan Scientific Journal. 2014; №2 (216): P. 8-11 (in Russian).

16. Varentsova VI, Varentsov VK, Bataev IA, Yusin SI. Effect of surface state of carbon fiber electrode on copper electroplating from sulfate solutions. Protection of Metals and Physical Chemistry of Surfaces. 2011; Vol. 47, № 1: pp. 43-47. 